\title{
RECONSTRUÇÃO PALEOAMBIENTAL DE UM DEPÓSITO SEDIMENTAR POR ANÁLISES MULTIPROXY, TURVO, ESTADO DO PARANÁ, BRASIL
}

\author{
GILIANE GESSICA RASBOLD \\ Pós-Graduação em Ecologia de Ambientais Aquáticos Continentais, Av. Colombo, 5790, Bloco G-90, 87020-900, \\ Maringá, Paraná, Brasil. grasbold@gmail.com \\ MAURO PAROLIN \\ Laboratório de Estudo Paleoambientais da FECILCAM, Rua Chafic Bader Maluf, 21, 87300-170, Campo Mourão, \\ Paraná, Brasil.mauroparolin@gmail.com
}

MARCELO GALEAZZI CAXAMBU

Herbário da Universidade Tecnológica Federal do Paraná,Via Rosalina Maria Dos Santos, 1233, 87301-899, Campo Mourão, Paraná, Brasil.mgcaxambu@yahoo.com.br

\begin{abstract}
PALEOENVIRONMENTAL RECONSTRUCTION OF A SEDIMENTARY DEPOSIT BY MULTIPROXY ANALYSIS, TURVO, STATE OF PARANÁ, BRAZIL. This research presents results of paleoenvironmental analysis that was achieved in a sedimentary record of $44 \mathrm{~cm}$ depth, recovered from a remnant of Mixed Rain Forest, located near the urban area in Turvo, Paraná. The record was dated $\left({ }^{14} \mathrm{C}\right)$ at two depths: 14,553 cal. years BP $(44 \mathrm{~cm})$ and 6.090 cal. years BP $(22 \mathrm{~cm})$. Besides the dates, five analyzes of $\delta^{13} \mathrm{C}$ were performed and increasing values from base $(-19.0 \%$ ) to top $(-22.9 \%)$ were obtained. Samples containing $20 \mathrm{~g}$ were extracted from the record on $2 \mathrm{~cm}$ intervals (22 samples); then the sediment dissolution $(2 \mathrm{~g})$ via chemical treatment was processed according to the protocol used in palynology with subsequent preparation of permanent slides ( $50 \mathrm{ul}$ material). The slides were observed under an optical microscope, where the count of 400 identifiable phytoliths by gap were determined and the concentration of phytoliths was estimated by random observation in 12 optical fields by gap (x 640). The adaptation indexes to drought (Iph\%), climate (Ic\%) and water stress (\% Fs) were calculated. Two paleoenvironmental areas were established: (i) Zone I, between 44 and $32 \mathrm{~cm}$ with a prevalence of drier conditions than current ones, that were evidenced by the increase in the rates of drought adaptation and water stress rates and mixture of $\mathrm{C}_{4}$ and $\mathrm{C}_{3}$ plants (pro $\mathrm{C}_{4}$ ); (ii) Zone II, from $30 \mathrm{~cm}$ to the top, characterized by a gradual increase in humidity, low rate of drought adaptation and impoverishment of the isotopic signal with $\mathrm{C}_{3}$ plants predominance, which was interpreted as an improvement in the moisture conditions and/or available high humidity in the soil. The interpretations corroborate studies for the south and west-central region in Brazil.
\end{abstract}

Key words: peatbog, phytoliths, carbon isotope.

RESUMO - A pesquisa apresenta resultados da análise paleoambiental realizada em um testemunho sedimentar de $44 \mathrm{~cm}$ de profundidade, recuperado de um remanescente de Floresta Ombrófila Mista, localizado próximo à área urbana de Turvo, Estado do Paraná. Foram obtidas idades pelo ${ }^{14} \mathrm{C}$ de 14.553 anos cal. AP a $44 \mathrm{~cm}$ e 6.090 anos cal. AP a $22 \mathrm{~cm}$, assim como cinco análises de $\delta^{13} \mathrm{C}$, com valores crescentes da base (-19,0\%) para o topo $(-22,9 \%$ ). Vinte e duas amostras, de $20 \mathrm{~g}$ cada, foram coletadas do testemunho, em intervalos de $2 \mathrm{~cm}$, para as analises de fitólitos. O sedimento foi submetido a tratamento químico $(1 \mathrm{~g})$, conforme protocolo utilizado na palinologia, com posterior confecção de lâminas permanentes $(50 \mu 1$ de material). As lâminas foram observadas ao microscópio óptico para quantificação de 400 fitólitos identificáveis por intervalo; estimouse a concentração de fitólitos de acordo com o procedimento da observação aleatória em 12 campos ópticos por intervalo (x 640). Foram calculados os índices de adaptação à aridez (Iph\%), climático (Ic\%) e de estresse hídrico (Fs\%). Tais análises permitiram estabelecer duas fases deposicionais: (i) Fase I, entre 44 e $32 \mathrm{~cm}$ com predomínio de condições paleoclimáticas mais secas que as atuais, evidenciadas pelo aumento nos índices de adaptação a aridez e índice de estresse hídrico e mistura de plantas $\mathrm{C}_{4}$ e $\mathrm{C}_{3}$ (pró $\mathrm{C}_{4}$ ); (ii) Fase II, de $30 \mathrm{~cm}$ ao topo, caracterizada por aumento gradativo da umidade, baixo índice de adaptação à aridez e empobrecimento do sinal isotópico com domínio de plantas $\mathrm{C}_{3}$, sugestivo de melhoria nas condições de umidade atmosférica e/ou alta umidade disponível no solo. As interpretações corroboram estudos realizados para a região sul e centro-oeste do Brasil.

Palavras-chave: turfeira, fitólitos, isótopos de carbono. 


\section{INTRODUÇÃO}

Para o estudo paleoambiental é necessário a utilização de ferramentas denominadas de indicadores proxy (esporos, espículas de esponjas, fitólitos, frústulas de diatomáceas entre outros), que neste caso, são registros locais, que podem ser interpretados utilizando princípios físicos ou biofísicos e tem o potencial de fornecer evidências de mudanças climáticas antes da existência de registros documentais, instrumentais ou históricos (IPCC, 2011). Para a realização destes estudos também são necessários locais propícios, e.g. ambientes lacustres e turfeiras, dadas suas condições de ativa sedimentação e de raros eventos erosivos (Suguio, 1999; Salgado-Labouriau, 1994).

Diante do exposto, e considerando a existência de depósitos sedimentares turfosos na região de Turvo, Estado do Paraná, utilizou-se como ferramenta proxy para interpretação paleoambiental da área em questão a qualificação/quantificação dos morfotipos de fitólitos, definidos como partículas de opala biogênica resultantes de processos físicos e biológicos que acontecem em algumas espécies de vegetais, encontrando-se em meio aos tecidos, dentro de algumas células (Rovner, 1988). Podem ocorrer em todas as estruturas da planta, como folhas, caules, raízes, inflorescências, frutos e sementes (Piperno, 1988; Mulhllonad, 1989). Estes corpos silicosos são liberados após a morte do vegetal, auxiliando na reconstrução do sistema vegetacional vigente em cada trecho de solo (Madella, 2007).

Verifica-se que os fitólitos, por sua constituição silicosa, resistem às condições ambientais oxidantes, diferente dos palinomorfos que são formados por esporopolenina e preservase apenas em condições anóxicas. Por isso, os fitólitos são proxy de extrema importância em estudos arqueológicos, especialmente na investigação agrícola de povos primitivos e respectiva domesticação de plantas (Piperno, 1991).

A produção de conhecimento sobre os fitólitos no Brasil, quando comparada a outros países, ainda é muito baixa. As primeiras pesquisas realizadas em território brasileiro datam da década de 1960 com os trabalhos de Sendulsky \& Labouriau (1966), Cavalcante (1968), Campos \& Labouriau (1969), mais recentemente com os trabalhos de Piperno \& Becker (1996), Alexandre et al. (1999), Calegari (2008), Medeanic et al. (2007; 2008), Santos et al. (2010), Rasbold et al. (2011); Coe et al. (2012), Raitz, (2012), Luz (2014), Monteiro (2015), entre outros.

Embora os fitólitos sejam característicos de gêneros ou de famílias especificas, alguns não tem significado taxonômico. Nas estruturas vegetativas de algumas plantas, especialmente as poáceas/gramíneas, ocorre uma infinidade de formas produzidas dentro da mesma espécie, por isso são necessárias avaliações multivariadas das assembleias para identificação correta (Piperno, 1988; Piperno, 1991).

As plantas, de acordo com o tipo, apresentam três tipos de ciclos fotossintéticos. O ciclo C3 é representado predominantemente por vegetação arbórea ( $85 \%$ de todas as espécies de plantas existentes). Plantas do ciclo C3 discriminam mais o ${ }^{13} \mathrm{C}$ durante o processo de fotossíntese que plantas do ciclo $\mathrm{C} 4$. Os valores de $\delta^{13} \mathrm{C}$ deste ciclo variam de $-22 \%$ a $-32 \%$, com média de -27\%o (Boutton, 1991).

As plantas do ciclo $\mathrm{C} 4$ incluem, predominantemente, poáceas/gramíneas de origem tropical e subtropical $(5 \%$ de todas as espécies de plantas). As gramíneas $\mathrm{C}_{4}$ estão relacionadas aos ambientes com altas temperaturas e alta luminosidade, cujos valores de $\delta^{13} \mathrm{C}$ variam de $-17 \%$ a $-9 \%$, com média de -13\%o (Boutton, 1991). Enquanto as plantas do ciclo CAM são representadas, geralmente, por suculentas e correspondem a $10 \%$ de todas as espécies, estas por sua vez, têm preferência por condições ambientais áridas e habitats epifíticos (Boutton, 1991). Estes sinais ficam registrados nos sedimentos após a morte das plantas e a análise de $\delta^{13} \mathrm{C}$ proporciona resultados confiáveis a respeito da troca de plantas $\mathrm{C}_{3}$ e $\mathrm{C}_{4}$ (Pessenda et al., 2005). Ressalta-se que em conjunto com os indicadores proxy é necessária a determinação cronológica dos sedimentos, que nesta pesquisa foi obtida via datação por ${ }^{14} \mathrm{C}$.

As mudanças ambientais ocorridas durante o Quaternário na região sul e em especial no Estado do Paraná, podem ser entendidas por meio de pesquisas paleoambientais que utilizam como ferramenta diversos microfósseis, grãos de pólen (e.g. Behling, 1997), espículas de esponja (e.g. Guerreiro et al., 2013), frústulas de diatomácea (e.g. Moro \& Foerstenberg, 1993) e fragmentos de carvão (e.g. Medeanic et al., 2008). No entanto, o número de publicações nessa linha é reduzido e a quantidade é ainda mais escassa quando utilizam-se os fitólitos (e.g. Monteiro, 2012; Calegari et al., 2013; Silva, 2013; Passos, 2014; Santos et al., 2014; Parolin et al., 2014; Luz, 2014). Observando a importância e a escassez de estudos de caráter paleoambiental, principalmente aqueles voltados para o período Quaternário no Estado do Paraná, a pesquisa traz novas e inéditas considerações sobre análise integrada de fitólitos e sinais isotópicos do depósito sedimentar mencionado desde o final do Pleistoceno.

\section{MATERIAL E MÉTODOS}

Aárea pesquisada (Figura 1) está localizada no município de Turvo, na região centro-sul do Estado do Paraná, Brasil. O estudo refere-se a um depósito sedimentar de característica turfosa. A coleta do material sedimentar foi realizada em remanescente de Floresta Ombrófila Mista, próximo à área urbana de Turvo (Figuras 1A-B).

O depósito está localizado nas coordenadas $25^{\circ} 02^{\prime} 58^{\prime \prime} \mathrm{S}$ e $51^{\circ} 33^{\prime} 21,54^{\prime \prime} \mathrm{O}$ à $1.044 \mathrm{~m}$ de altitude. Foi coletado um testemunho sedimentar de $44 \mathrm{~cm}$ de profundidade, via inserção de tubo de alumínio de $10 \mathrm{~cm}$ de diâmetro (Figura 1A) com auxílio de marreta. Observou-se que o sedimento turfoso está em contato abrupto com a rocha basáltica da Formação Serra Geral (MINEROPAR, 2006). Em laboratório, o tubo foi aberto com auxílio de serra circular e coletadas 22 amostras de 20 g sistematicamente a cada 2 $\mathrm{cm}$. Todas as amostras sedimentares foram secas em estufa $\left(70^{\circ} \mathrm{C} / 24 \mathrm{~h}\right)$.

Após secagem, encaminhou-se $5 \mathrm{~g}$ de sedimentos de dois intervalos para determinação da cronologia via datação 


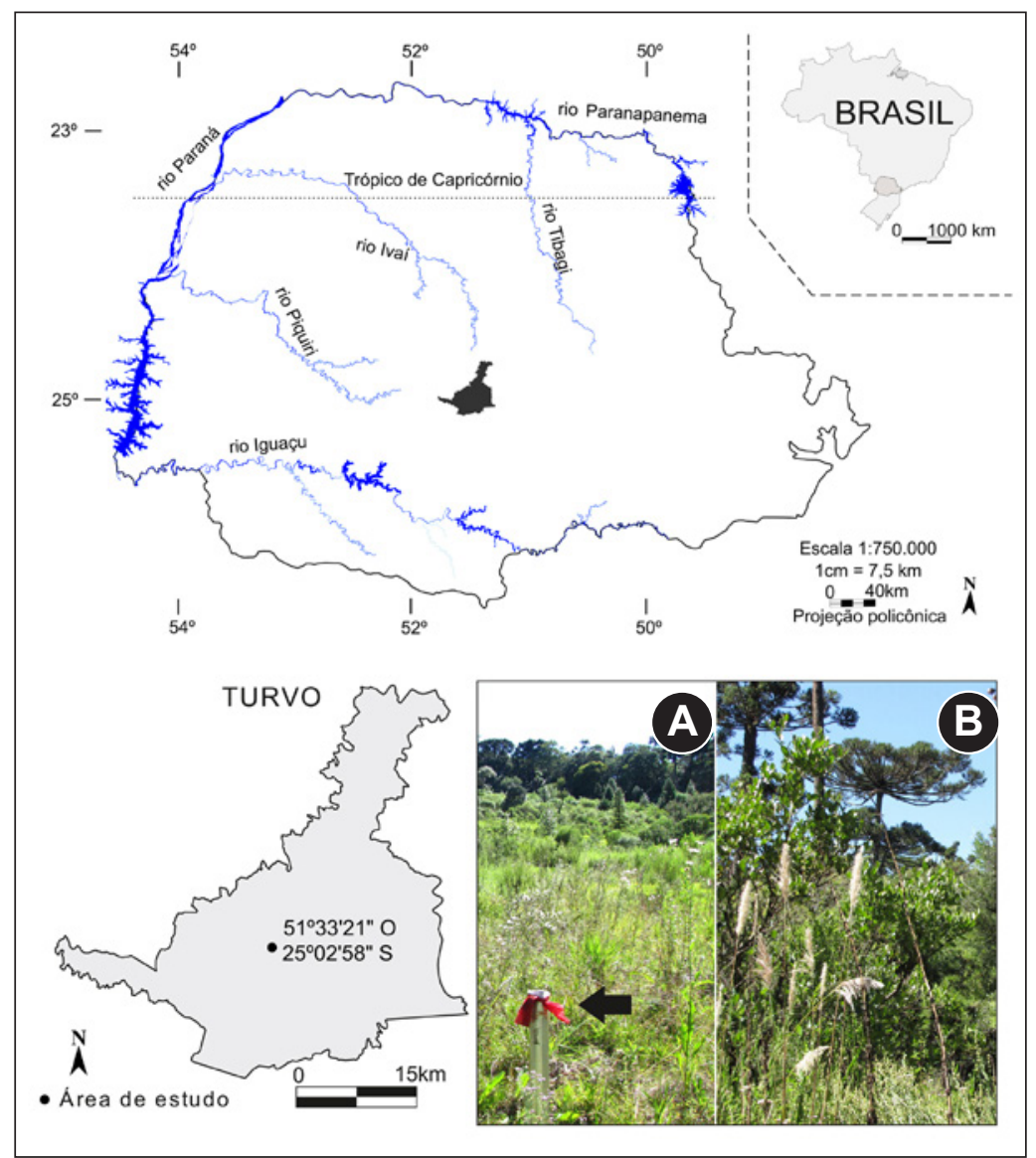

Figura 1. Localização da área de estudo, Turvo, Estado do Paraná. A, tubo de alumínio; B, entorno do local de coleta.

Figure 1. Location of study area, Turvo, State of Paraná. A, aluminum tube with core; B, surroundings of the collection site.

radiocarbônica (20-22 cm e 42-44 cm) e $5 \mathrm{~g}$ de cinco intervalos para a determinação da composição isotópica $\left(\delta^{13} \mathrm{C}\right.$ - expressa em por mil (\%) com desvio padrão de $\pm 0.2 \%$, medido com respeito ao padrão VPDB) nos intervalos de 0-2, 10-12, 20-22, 32-34 e 42-44 cm. As análises foram efetuadas no Center for Applied Isotope Studies, University of Georgia.

A análise da razão entre ${ }^{13} \mathrm{C} /{ }^{12} \mathrm{C}$ do carbono orgânico de sedimentos possibilita a distinção de plantas com ciclo fotossintético $\mathrm{C}_{3}$ e $\mathrm{C}_{4}$, o entendimento da composição paleovegetacional e as alterações nas comunidades ao longo do tempo (Boutton, 1991; Pessenda et al., 1996a,b; 2005). A utilização dos isótopos estáveis do carbono aliadas a identificação de morfotipos de fitólitos podem fornecer evidências concisas a respeito da dinâmica da vegetação.

O tratamento químico para extração dos fitólitos foi realizado seguindo metodologia de Faegri \& Iversen (1989): (i) separação de $5 \mathrm{~cm}^{3}$ do material coletado em béqueres; (ii) adição de ácido clorídrico (concentração 10\%) às amostras, para eliminação de carbonatos; (iii) fervura das amostras com hidróxido de potássio a $10 \%$; (iv) adição de solução aquosa de cloreto de zinco densidade $2,4 \mathrm{~g} / \mathrm{cm}^{3}$; (v) centrifugou-se uma vez as amostras (500 RPM por 3 min.); (vi) o sobrenadante contendo as partículas com densidade inferior à $2,4 \mathrm{~g} / \mathrm{cm}^{3}$ foi retirado e posteriormente centrifugado (500 RPM por 3 minutos) com água destilada até eliminação total do cloreto de zinco. Após o tratamento químico, foi adicionado $50 \mu 1$ de material em lâminas, que após secas, foram recobertas com Entellan $^{\circledR}$ e lamínula. As lâminas encontram-se depositadas no Laboratório de Estudos Paleoambientais da Faculdade Estadual de Ciências e Letras de Campo Mourão (UEPRLepafe) (Cód. L. 206; 207; 208. C.19).

Para as análises qualitativas e quantitativas da assembleia de fitólitos analisou-se em microscópio óptico em aumento de $640 \mathrm{x}$, duas lâminas de cada intervalo, foram contados 200 fitólitos identificáveis por lâmina. Para identificação utilizou-se da vasta literatura a respeito (Piperno, 1988; 2006; Piperno \& Pearsall, 1998; Madella et. al., 2005; Twiss et al., 1969; Raitz, 2012), além da coleção de referência do Lepafe. Os fitólitos foram classificados de acordo com sua morfologia, utilizando como base para a nomenclatura o trabalho de Madella et al. (2005). Paralelamente à contagem, foram realizadas microfotografias em aumento de $640 \mathrm{x}$ dos morfotipos característicos.

Para a análise quantitativa da concentração de fitólitos, realizou-se a contagem via observação de 12 campos de microscopia (quatro campos/lâmina, em três lâminas) selecionados aleatoriamente em aumento de $640 \mathrm{x}$. Posteriormente foi realizada a média aritmética das concentrações, resultando na concentração média de fitólitos por campo de microscopia. 
Procedeu-se o cálculo dos índices fitolíticos, que visam refinar e auxiliar a interpretação paleoambiental com base nos morfotipos característicos de subfamílias de Poaceae (Bremond, 2003; Bremond et al., 2005). Estes consistem em: Índice de adaptação à aridez - Iph. (Diéster-Haass et al., 1973). Faz relação entre morfotipos característicos de gramíneas $\mathrm{C}_{4}$ adaptadas a condições mais e menos áridas. Para o cálculo utiliza-se a equação Iph $(\%)=[($ saddle $) /$ (saddle + bilobate + cross) $] * 100$, onde valores elevados de Iph indicam condições mais secas e valores menores indicam uma condição de maior umidade ou umidade presente no solo; Índice climático - Ic. (Twiss, 1992; Bremond, 2003). Tratase da relação entre morfotipos representativos de gramíneas $\mathrm{C}_{3} / \mathrm{C}_{4}$, sendo a equação representada por $I c(\%)=[($ rondel $) /$ (rondel + bilobate + cross + saddle $)]^{* 100}$, onde valores elevados indicam predomínio de gramíneas adaptadas a condições mais frias, em relação às gramíneas adaptadas a climas mais quentes;

Índice de estresse hídrico - Fs. (Bremond et al., 2005). Faz relação entre os morfotipos cuneiform bulliform (presente em gramíneas que foram submetidas a elevadas taxas de evapotranspiração) e à soma dos morfotipos short cells (bilobate, cross, saddle e rondel, característicos de Poaceae), a equação que define este índice é expressa por $F_{s}(\%)=$ $[$ (cuneiform)/(cuneiform + bilobate + cross + saddle + rondel)]*100, valores elevados são indicativos de condições de maior evapotranspiração das plantas.

As fases de deposição sedimentar foram diferenciadas pelo programa CONISS ${ }^{\circledR}$ (Grimm, 1987).

\section{RESULTADOS E DISCUSSÃO}

Tendo em vista a pequena espessura do testemunho, sua característica turfosa e seu contato abrupto com a base rochosa, pode-se afirmar que a taxa de sedimentação foi lenta. As datações indicaram idade pleistocena na base testemunho (14.553 anos cal. AP aos 44-42 cm) com taxa de deposição de aproximadamente $0,0026 \mathrm{~cm} /$ ano e holocena no meio $(6.090$ anos cal. AP aos $22-20 \mathrm{~cm}$ ), onde nota-se incremento de $\sim 28 \%$ na taxa de deposição sedimentar, $0,0036 \mathrm{~cm} /$ ano (Tabela 1).

Os valores de $\delta^{13} \mathrm{C}$ são enriquecidos próximo a base e mais empobrecidos próximo ao topo (Tabela 1). O sinal isotópico da base indica uma mistura entre plantas de ciclo fotossintético $\mathrm{C}_{3} \mathrm{e} \mathrm{C}_{4}$, caracterizando vegetação mais aberta. Já em direção ao topo verifica-se a tendência para plantas $\mathrm{C}_{3}$ evidenciando maior densidade vegetacional.

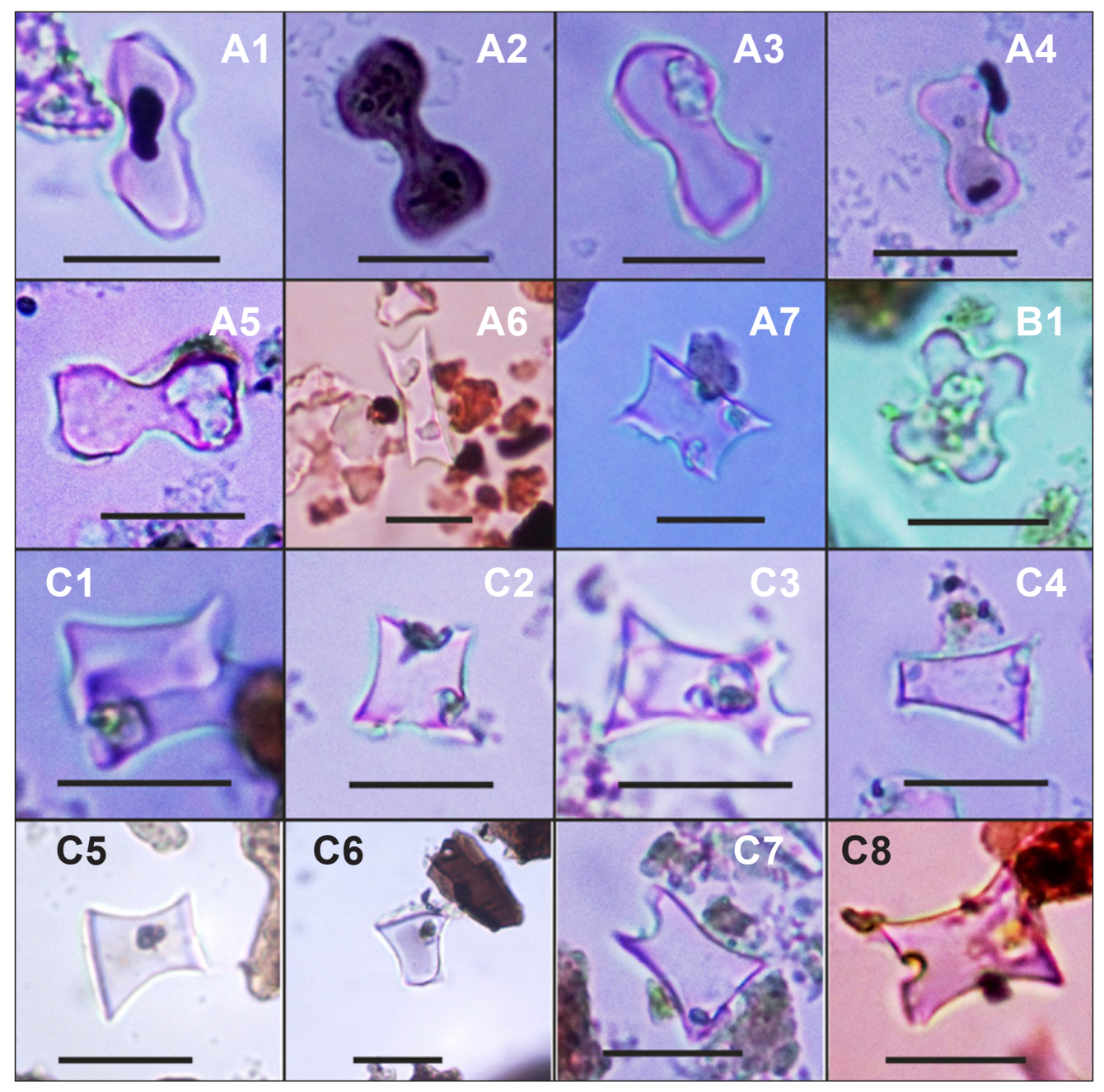

Figura 2/Figure 2. Morfotipos de fitólitos/phytoliths morphotypes. A1-A7, Bilobate; B1, Cross; C1-C8, Rondel. Escalas/Scale bars = 17 $\mu$ m. 
Tabela 1. Resultados isotópicos.

Table 1. Isotopic results.

\begin{tabular}{cccc}
\hline Profundidade $(\mathrm{cm})$ & $\delta^{13} \mathrm{C} \%$ o & ${ }^{14} \mathrm{C}$ cal. anos AP & Taxa de sedimentação (cm/ano) \\
\hline $0-3$ & $-22,9$ & & 0,0036 \\
$10-12$ & -21.5 & - & \\
$20-22$ & -19.0 & 6.090 & 0,0026 \\
$32-34$ & -19.3 & - & 14.553 \\
$42-44$ & -19.0 & & 0 \\
\hline
\end{tabular}

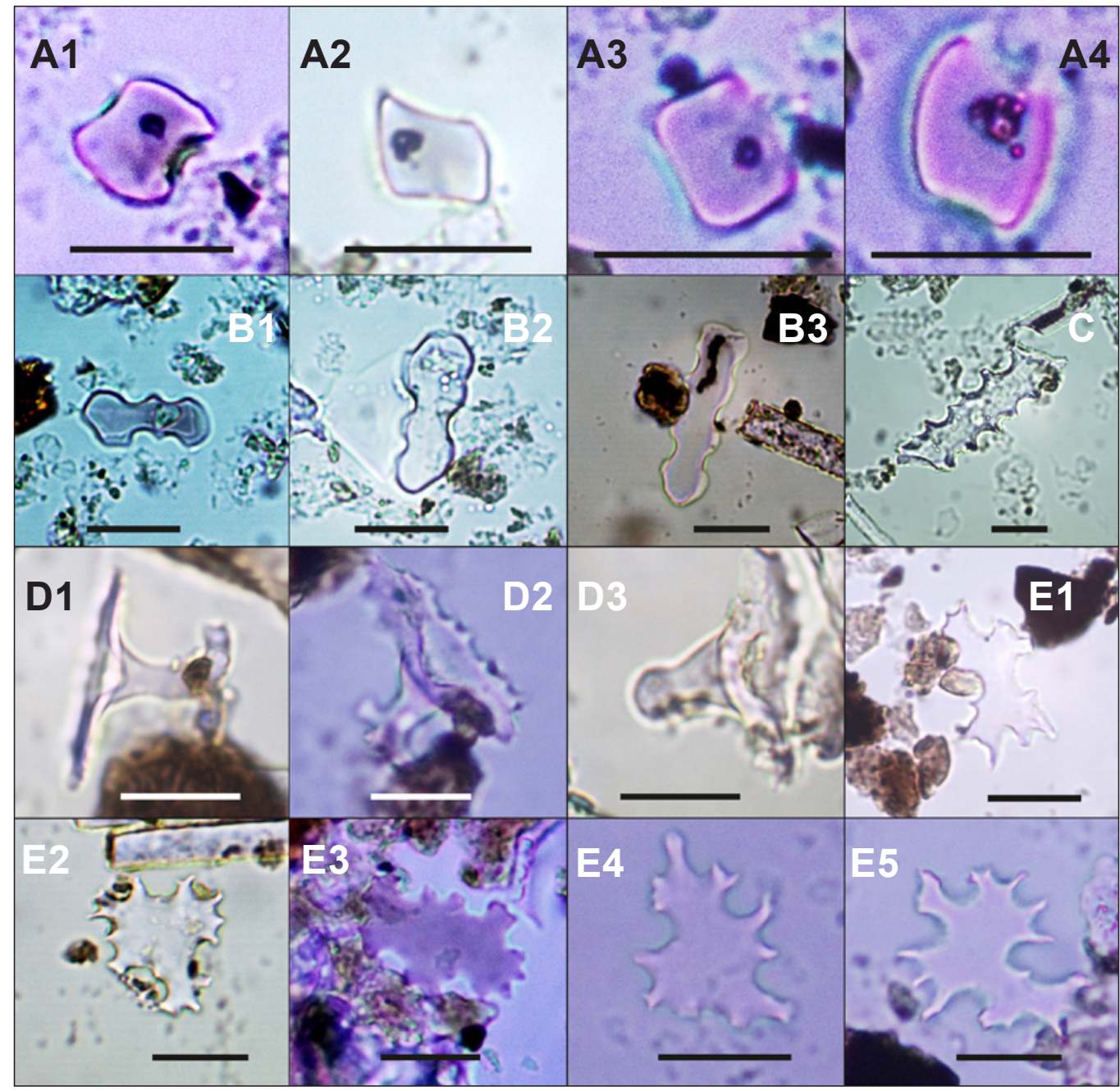

Figura 3/Figure 3. Morfotipos de fitólitos/phytoliths morphotypes. A1-A4, Saddle; B1-B3, Cylindric polylobate; C, Elongate echinate; D1-D3, Cone-shape; E1-E5, Irregular. Escalas/Scale bars $=17 \mu \mathrm{m}$.

Identificou-se 23 morfologias de fitólitos que variaram ao longo do perfil sedimentar (Figuras 2-5). Dentre estas destacam-se as denominadas short-cells produzidas por gramíneas da família Poaceae (Bilobate, Figuras 2A1A7; Cross, Figura 2B1; Rondel, Figuras 2C1-C8; Saddle, Figuras 3A1-A4), algumas morfologias que não apresentam padrão taxonômico específico, mas que são produzidas por gramíneas (Cylindrical polylobate, Figuras 3B1-B3; Elongate echinate, Figura 3C; Elongate psilate, Figuras 4A1-A2; Cylindric sulcate tracheid, Figuras 4B1-B2; Cuneiform bulliform, Figuras 4C1-C4; Brachiform, Figuras 4D1-D4; Parallepipedal bulliform, Figuras 4E1-E4), poáceas da família
Cyperaceae (Cone-shape, Figuras 3D1-D3) e uma morfologia a qual não se conseguiu atribuir a alguma família botânica (Irregular, Figuras 3E1-E5).

A morfologia predominante foi elongate psilate, no entanto, não possui valor taxonômico, pois é produzida na epiderme de todas as gramíneas (Bremond, 2003). As morfologias bilobate, cross, sadlle e rondel estão associadas a diferentes tipos de subfamílias de Poaceae e podem mostrar mudanças de vegetação. Como a ocorrência destas morfologias foram superiores a $5 \%$ em todos os intervalos, foi possível estabelecer os índices (Iph, Ic e Fs) (Coe, 2009) em todas as sequências (Figura 5). As morfologias bulliform são tratadas na literatura 


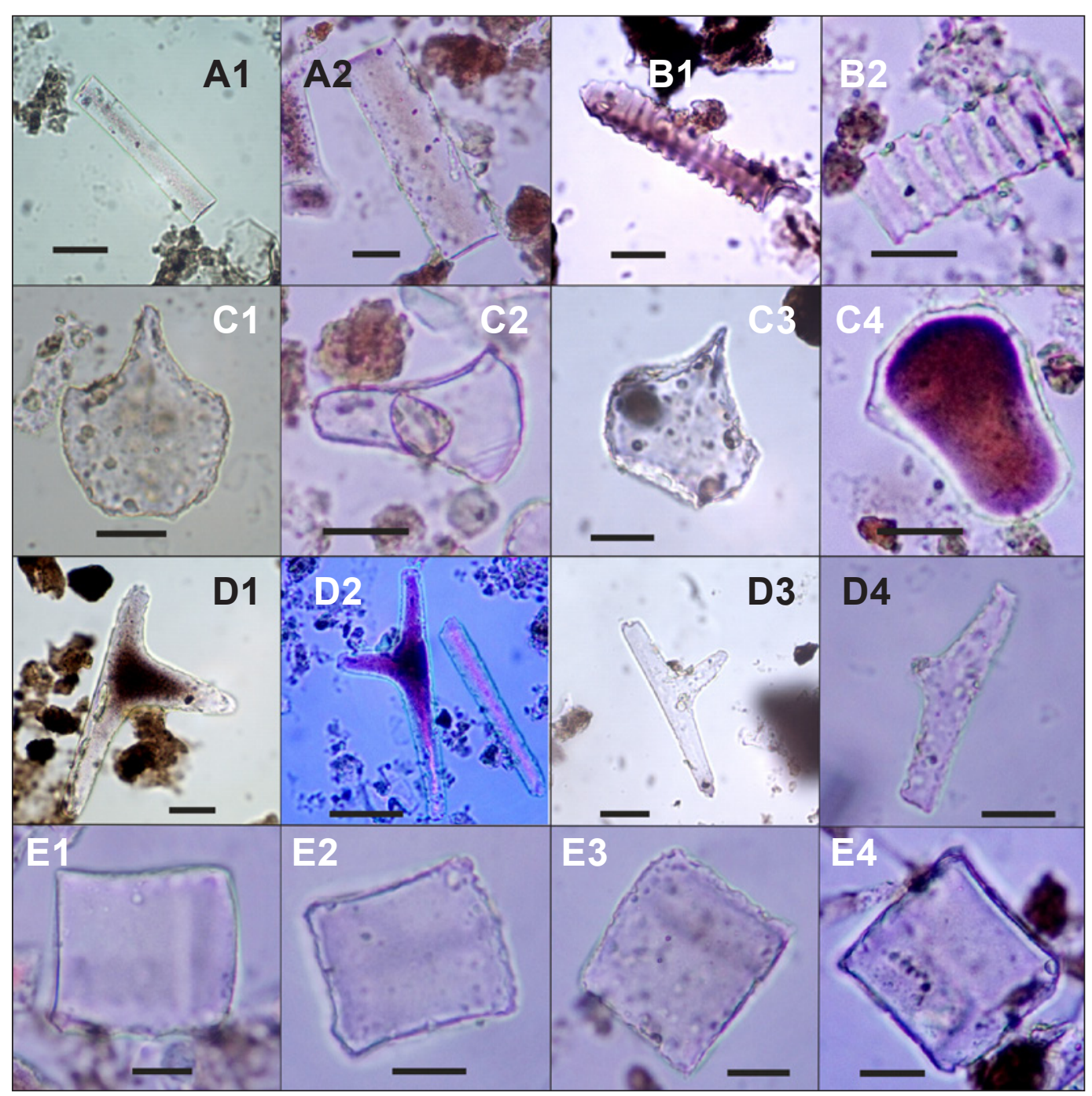

Figura 4/Figure 4. Morfotipos de fitólitos/phytoliths morphotypes. A1-A2, Elongate psilate; B1-B2, Cylindrical sulcate tracheid; C1-C4, Cuneiform bulliform; D1-D4, Brachiform; E1-E4, Parallepipedal bulliform. Escala/Scale bars $=17 \mu \mathrm{m}$.

como indicadores de estresse hídrico, relacionando a produção destas à potencialização do processo de evapotranspiração de gramíneas (Parry \& Smithson, 1958; Sangster \& Parry, 1969; Bremond, 2003; Bremond et al., 2005). No perfil estão associadas a este processo e se comportam de maneira similar ao longo das sequências analisadas.

Foi observada a presença de um morfotipo, sem similaridade com aqueles já descritos e disponíveis na literatura, aqui denominado como irregular, e, portanto, não foi possível até o presente momento relacioná-lo a algum grupo botânico. Este morfotipo é encontrado mais frequente entre $28-0 \mathrm{~cm}$.

Com software CONISS ${ }^{\circledR}$ (Grimm, 1987) foi possível estabelecer duas fases de deposição sedimentar:

\section{Fase I. 44-32 cm (14.553 anos cal. AP 44-42 cm)}

Nesta fase a concentração de fitólitos é baixa e constante (0 a 15 grãos/campo de microscopia) (Figura 5). Foi observado um pico $(33,6 \%)$ dos morfotipos bulliform (cuneiform e parallepipedal) no intervalo entre $36-34 \mathrm{~cm} \mathrm{e}$, por consequência, o índice $F$ s igual a 51,6\% indica elevado estresse hídrico.
Os valores de $I p h$ são elevados (> 50\%) (intervalos 3032 e 40-42 cm) e caracterizam elevada adaptação à aridez. Bremond et al. (2005), em trabalho realizado na região leste da África, mostraram que condições climáticas quentes e secas coincidiam com a elevação deste índice.

Os sinais isotópicos dos intervalos $44-42 \mathrm{~cm}\left(\delta^{13} \mathrm{C}-19.0 \%\right.$ ) e $34-32 \mathrm{~cm}\left(\delta^{13} \mathrm{C}-19.3 \%\right)$ são similares e pode-se inferir que no decorrer desta fase a vegetação era mista incluindo plantas $\mathrm{C}_{3} \mathrm{e}_{4}$, com predomínio de $\mathrm{C}_{4}$ (Figura 5). Essa situação sugere condições climáticas mais secas que as atuais no final do Pleistoceno e no Holoceno inferior.

Tais interpretações corroboram com aquelas obtidas na região sul e centro-oeste do Brasil, tais como: (i) Luz (2014) quantificou/qualificou fitólitos recuperados de sedimentos turfosos e constatou uma fase eminentemente mais seca que a atual na transição do Pleistoceno superior/ Holoceno, há 16.010 anos cal. AP na planície aluvial do rio Ranchinho, Campo Mourão; (ii) Pessenda et al. (2004), com base na determinação de valores de $\delta^{13} \mathrm{C}$, em testemunhos localizados no município de Londrina, relatam a ocorrência de clima mais seco entre 11.000-8.000 anos AP; (iii) Parolin 


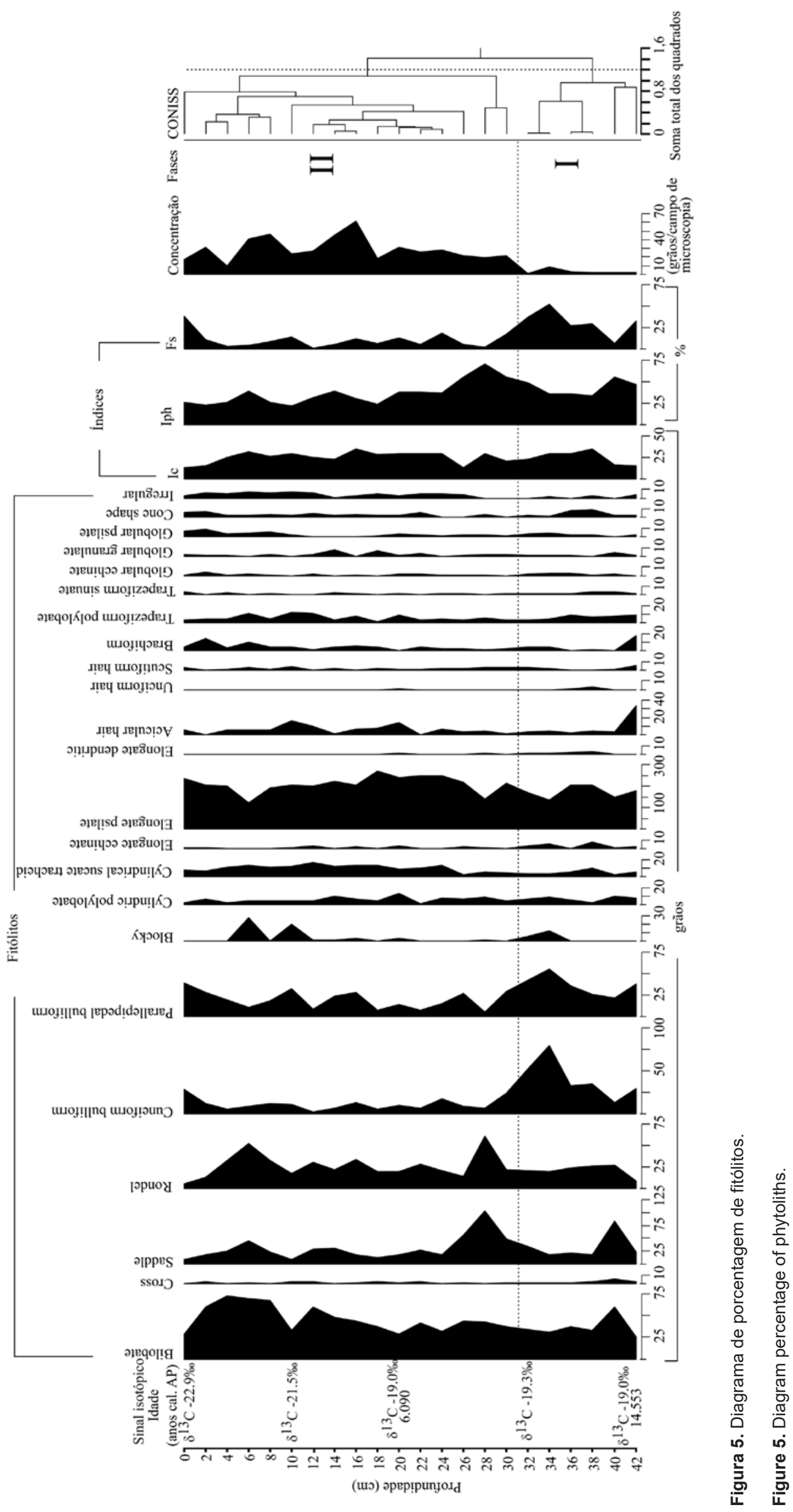


et al. (2006) desenvolveram estudos palinológicos em lagoas no município de Taquarussu, no Estado de Mato Grosso do Sul, e observaram um período de recrudescência climática entre 11.570 e 9.710 anos AP; (iv) Behling (1997) analisou o conteúdo palinológico de um testemunho localizado nos Campos Gerais, no Estado do Paraná e concluiu que no período de 12.480 à 9.660 anos $\mathrm{AP}$ ocorreu um clima mais frio e seco, com geadas severas; (v) Ledru et al. (1998) correlacionaram registros de grãos de pólen de onze localidades brasileiras representadas pelos principais tipos de vegetação, as análises forneceram subsídios para a interpretação de ambientes com condições frias e secas no período ente 10.000 e 7.000 anos AP.

\section{Fase II. 30-0 cm (6.090 anos cal. AP. 22-20cm)}

Ocorre aumento gradativo da concentração de fitólitos (Figura 5), atingindo o pico máximo de deposição no intervalo de 18-16 cm, no qual foi possível a observação de $\sim 70$ grãos/ campo de microscopia. Verificou-se nessa fase menor estresse hídrico (diminuição da ocorrência do morfotipo bulliform), bem como pico de deposição dos morfotipos saddle e rondel no intervalo de $28-30 \mathrm{~cm}$, seguido pela redução destes e aumento gradativo do morfotipo bilobate.

O Iph diminui $(<39 \%)$, podendo ser associado com clima mais úmido e/ou alta umidade disponível no solo (Barboni et al., 1999; Bremond et al., 2005). Houve aumento gradativo dos morfotipos globular echinate (Arecaceae - Piperno, 2006; Runge, 1999; Mercader et al., 2009 e Bromeliaceae - Piperno, 1985, 2006; Bremond et al., 2005), globular granulate e psilate (monocotilêdonias, dicotiledônias e coníferas - Carnelli et al., 2004), acompanhados do morfotipo coneshape (Cyperaceae - Ollendorf, 1992; Barboni et al., 1999; Honaine et al., 2009) e o morfotipo irregular. O índice de estresse hídrico $(F s)$ diminui. Os dados evidenciam condições de vegetação mais densa que a fase anterior, podendo ser arborizada (Figura 6).

Os sinais isotópicos desta zona apresentam empobrecimento próximo do topo, nos intervalos de $12-10 \mathrm{~cm}-21.5 \%$ e de $0-2$ $\mathrm{cm}-22.9 \%$. Estes resultados indicam predomínio de plantas $\mathrm{C}_{3}$, provavelmente resultantes de condições mais úmidas a partir do Holoceno médio.

As interpretações desta pesquisa corroboram estudos realizados na região sul e centro-oeste do Brasil para o Holoceno: (i) Luz (2014), evidenciou incremento de umidade a partir de 7.280 anos cal. AP, para um testemunho do rio Ranchinho em Campo Mourão; (ii) Santos et al. (2014), quantificaram e qualificaram os fitólitos presentes em solo de área florestal no município de Douradina, no Estado do Paraná e constataram que as condições climáticas eram mais úmidas que as atuais, com maior cobertura arbórea, há pelo menos 5.963 anos cal. AP; (iii) Parolin et al. (2006) observaram uma fase mais úmida, nos depósitos estudados em Taquarussu, no Estado de Mato Grosso do Sul, há 7.500 e 3.500 anos AP; (iv) Guerreiro (2011) sugeriu que a partir de 3.220 anos AP, na região do alto Tibagi, Campos Gerais, no Estado do Paraná, a deposição teria ocorrido em condições ambientais pantanosas e climáticas úmidas, embora ligeiramente mais secas que as
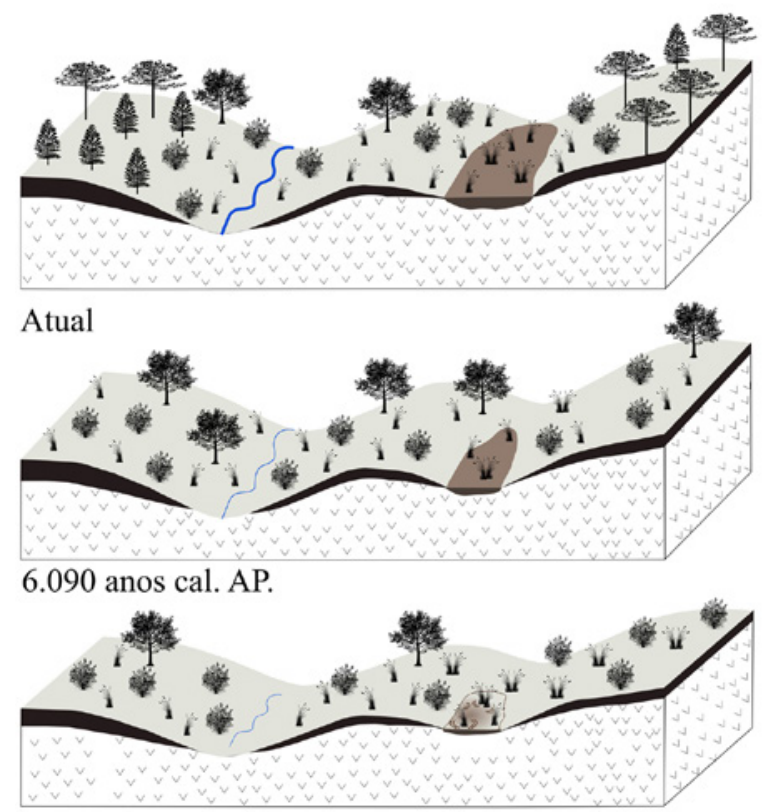

14.553 anos cal. AP.

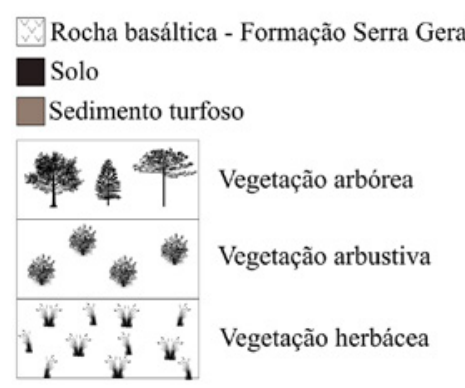

Figura 6. Bloco diagrama demonstrando a variação da vegetação que provavelmente produziu a Turfa.

Figure 6. Block-diagram showing the variation of vegetation that probably produced peat Turfa Turvo.

atuais; (v) Ledru et al. (1998) mostraram aumento na umidade atmosférica entre 7.000 e 4.000 anos AP e sugeriram que a partir de 4.000 anos AP estabeleceram-se as condições climáticas modernas.

\section{CONCLUSÃO}

Embora os estudos de fitólitos tenham sua concentração na área arqueológica, o interesse por essa microestrutura para o entendimento de questões paleoambientais e ambientais tem aumentado nas últimas décadas, principalmente em estudos de caráter ambiental. Nesse sentido, os resultados obtidos pelo presente estudo reforçam o uso desta ferramenta em depósitos sedimentares.

Os sedimentos estudados têm sua origem relacionada, cronologicamente, ao final do Pleistoceno. Os fitólitos apresentaram boa preservação e, por isso, foi possível qualificá-los e quantificá-los, permitindo que fossem elaborados diagramas e consequentemente estabelecidas duas fases de deposição. A primeira tem início há 14.553 anos cal. AP sob condições climáticas, sugestivamente mais secas, evidenciadas por altos valores do índice de adaptação 
a aridez, de estresse hídrico e sinal isotópico indicando mistura de plantas $\mathrm{C}_{3}$ e $\mathrm{C}_{4}$ pró $\mathrm{C}_{4}$. A segunda fase data do Holoceno médio, 6.090 anos cal. AP e é caracterizada por empobrecimento do sinal isotópico, diminuição gradativa dos índices de adaptação a aridez e estresse hídrico, sugerindo clima mais úmido e/ou alta umidade disponível no solo.

\section{AGRADECIMENTOS}

Os autores agradecem ao CNPq (processo 472.496/20115). A primeira autora agradece à Coordenação de Aperfeiçoamento de Pessoal de Nível Superior (CAPES) pela bolsa e ao PROEX/CAPES. O segundo autor agradece à Fundação Araucária pela bolsa de produtividade.

\section{REFERENNCIAS}

Alexandre, A.; Meunier, J.D.; Mariotti, A. \& Soubies, F. 1999. Late Holocene Phytolith and Carbon-Isotope Record from a Latosol at Salitre, South-Central Brazil. Quaternary Research, 51:187194. doi:10.1006/qres.1998.2027

Barboni, D.; Bonnefille, R.; Alexandre, A. \& Meunier, J.D. 1999. Phytoliths as paleoenvironmental indicators, West Side Middle Awash Valley Ethiopia. Palaeogeography, Palaeoclimatology, Palaeoecology, 152:87-100. doi:10.1016/ S0031-0182(99)00045-0

Behling, H. 1997. Late Quaternary vegetation, climate and fire history of the Araucaria forest and campos region from Serra Campos Gerais, Paraná State (South Brazil). Review of Palaeobotany and Palynology, 97:109-121. doi:10.1016/S00346667(96)00065-6

Boutton, T.W. 1991. Stable carbon isotope rations of natural materials: II. Atmospheric, terrestrial, marine, and freshwater environmental. In: D.C. Coleman \& B. Fry (eds.) Carbon Isotope Techniques, Academic Press, p. 173-185.

Bremond, L. 2003. Calibration des fonctions de transfert entre assemblages phytolithiques, structure des vegetations et variables bioclimatiques actuelles, pour l'integration de la ynamique des biomes herbaces dans les modeles de vegetation. Géologie appliquée, Universite de Droit, d'Économie et des Sciences D'Aix-Marseille, Tese de doutorado, $197 \mathrm{p}$.

Bremond, L.; Alexandre, A.; Peyron, O. \& Guiot, J. 2005. Grass water stress estimated from phytoliths in West Africa. Journal of Biogeography, 32:311-327. doi:10.1111/j.13652699.2004.01162.x

Calegari, M.R. 2008. Ocorrência e significado paleoambiental do horizonte A húmico em latossolos. Programa de Pós-Graduação em Agronomia, Universidade de São Paulo, Escola Superior de Agricultura "Luiz de Queiroz", Tese de doutorado, 259 p.

Calegari, M.R.; Madella, M.; Vidal-Torrado, P.; Pessenda, L.C.R. \& Marques, F.A. 2013. Combining phytoliths and $\delta^{13} \mathrm{C}$ matter in Holocene palaeoenvironmental studies of tropical soils: an example of an Oxisol in Brazil. Quaternary International, 287:47-55. doi:10.1016/j.quaint.2011.11.012

Campos, A.C. \& Labouriau, L.G. 1969. Corpos silicosos de gramíneas dos Cerrados. II. Pesquisa Agropecuária Brasileira, 4:143-151.

Carnelli, A.L.; Theurillatb, J.P. \& Madella, M. 2004. Phytolith types and type-frequencies in subalpine-alpine plant species of the European Alps. Review of Palaeobotany and Palynology, 129:39-65. doi:10.1016/j.revpalbo.2003.11.002
Cavalcante, P.B. 1968. Contribuição ao estudo dos corpos silicosos das gramíneas Amazônicas I. Panicoideae (Melinideae, Andropogoneae e Tripsaceae). Botânica, 80:1-11.

Coe, H.H.G. 2009. Fitólitos como indicadores de mudanças na vegetação xeromórfica da região de Búzios/Cabo Frio, $R J$, durante o Quaternário. Programa de Pós-Graduação em Geologia e Geofísica Marinha, Universidade Federal Fluminense, Tese de doutorado, 340 p.

Coe, H.H.G.; Chueng, K.F. \& Gomes, J.G. 2012. Reconstituições da vegetação e inferências de paleoclimas através da utilização dos indicadores fitólitos e isótopos de carbono - exemplos de estudos no Brasil. Revista Geonorte, 1:248-261.

Diester-Haass, L.; Schrader, H.J. \& Thiede, J. 1973. Sedimentological and paleoclimatological investigations of two pelagic ooze cores off Cape Barbas, North-West Africa. Meteor Forshungergebnisse, C16:19-66.

Faegri, K. \& Iversen, J. 1989. Textbook of pollen analysis. $4^{\mathrm{a}} \mathrm{ed}$. New York, John Wiley, 295 p.

Grimm, E. 1987. CONISS: A Fortran 77 Program for stratigraphically constrained cluster analysis by the method of incremental sum of squares. Computers \& Geosciences, 13:13-35.

Guerreiro, R.L. 2011. Paleoambientes holocênicos da planície do Alto Tibagi, Campos Gerais, Sudeste do Estado do Paraná. Programa de Pós-Graduação em Geografia, Universidade Estadual de Maringá, Dissertação de mestrado, 88 p.

Guerreiro, R.L.; Stevaux, J.C.; Parolin, M. \& Assine, M.L.A. 2013. Late Pleistocene and Holocene paleoenvironments in ponds and alluvial sediments of Upper Paraná river, Brazil. Revista Brasileira de Paleontologia, 16:39-46. doi:10.4072/ rbp.2013.1.03.

Honaine, M.F.; Zucol, A.F. \& Osterrieth, M. 2009. Phytolith analysis of Cyperaceae from the Pampean region, Argentina. Australian Journal of Botany, 57:512-523. doi:10.1071/bt09041

IPCC. 2011. Intergovernmental Panel on Climate Change. Palaeoclimate proxy indicators. Disponível em http://ipcc.ch/ ipccreports/tar/wg1/068.htm; acessado em 28/06/ 2011.

Ledru, M.P.; Salgado Laboriau, M.L. \& Lorscheitter, M.L. 1998. Vegetation dynamics in Brazil during the last 10,000 yr BP. Review of Palaeobotany and Palynology, 99:131-142. doi:10.1016/s0034-6667(97)00049-3

Luz, L.D. 2014. Aspectos paleoambientais do Quaternário Superior na Região de Campo Mourão, Paraná. Programa de PósGraduação em Geografia, Universidade Estadual de Maringá, Dissertação de mestrado, 126 p.

Madella, M. 2007. Phytoliths: a terrestrial botanical dataset for palaeoenvironmental reconstructions. Geophysical Research Abstracts, 9:06057.

Madella, M.; Alexandre, A. \& Ball, T. 2005. International code for phytolith nomenclature 1.0. Annals of Botany, 96:253-260. doi:10.1093/aob/mci172

Medeanic, S.; Cordazzo, C.V.; Corrêa, I.C.S. \& Mirlean, N. 2008. Os fitólitos em gramíneas de dunas do extremo Sul do Brasil: variabilidade morfológica e importância nas reconstruções paleoambientais costeiras. Gravel, 6:1-14.

Medeanic, S.; Dillenburg, S.R. \& Weschenfelder, J. 2007. Palinomorfos nos sedimentos de fundo de laguna dos Patos, RS: aplicação nas reconstruções paleoambientais. Gravel, 5:89-102.

Mercader, J.; Bennett, T.; Esselmont, C.; Simpson, S. \& Walde, D. 2009. Phytoliths in woody plants from the Miombo woodlands of Mozambique. Annals of Botany, 104:91-113. doi:10.1093/ aob/mcp097 
MINEROPAR. 2006. Atlas geomorfológico do Paraná. Disponível em http://www.mineropar.pr.gov.br/modules/conteudo/conteudo. php? conteudo=133; acessado em 14/07/2013.

Monteiro, M.R. 2012. Paleoclimatologia do Holoceno Médio indicadas por fitólitos nos Campos Gerais. Engenharia Ambiental, Universidade Tecnológica Federal do Paraná, Monografia de conclusão de curso, 60 p.

Monteiro, M.R. 2015. Análise da composição fitolítica da serapilheira e solo como indicador de alterações ambientais em diferentes estratos arbóreos no Paraná. Programa de PósGraduação em Geografia, Universidade Estadual de Maringá, Dissertação de mestrado, 101 p.

Moro, R.S. \& Fürstenberger, C.B. 1993. Diatomáceas (Bacillariophyceae) da Lagoa Dourada (Parque Estadual de Vila Velha), Paraná, Brasil. Acta Biológica Paranaense, 22:15-30.

Mulhllonad, S.C. 1989. Phytolith shape frequencies in North Dakota grasses: a comparison to general patterns. Journal of Archeological Science, 16:489-511. doi:10.1016/03054403(89)90070-8

Ollendorf, A.L. 1992. Toward a classification scheme of sedge (Cyperaceae) phytolith. In: G. Rapp Jr. \& S.C. Mulholland (eds.) Phytolith Systematics. Emerging Issues, Plenum Press, p. 91-111.

Parolin, M.; Medeanic, S. \& Stevaux, J.C. 2006. Registros palinológicos e mudanças ambientais durante o Holoceno de Taquarussu (MS). Revista Brasileira de Paleontologia, 9:137-148.

Parolin, M.; Rasbold, G.G. \& Pessenda, L.C.R. 2014. Paleoenvironmental conditions of Campos Gerais, Paraná, since the Late Pleistocene, based on phytoliths and C and $\mathrm{N}$ Isotopes. In: H.H.G. Coe \& M. Osterrieth (eds.) Sinthesis of Some Phytolith Studies in South America, Nova Science Publishers, p. 149-170.

Parry, W.D. \& Smithson, F. 1958. Silicification of bulliform cells in grasses. Nature, 181:1549-1550. doi:10.1038/1811549b0

Passos, J.R. 2014. Caracterização das Linhas de Pedra, na Bacia Hidrográfica do Rio Bananas, Guarapuava-Paraná. Programa de Pós-Graduação em Geografia, Universidade Estadual do Centro-Oeste do Paraná, Dissertação de mestrado, 83 p.

Pessenda, L.C.R.; Aravena, R.; Melfi, A.J.; Telles, E.C.C.; Boulet, R.G.L.; Valencia, E.P.E. \& Tomazello, M. 1996a. The use of carbon isotopes $(\mathrm{C}-13, \mathrm{C}-14)$ in soil to evaluate vegetation changes during the Holocene in Central Brazil. Radiocarbon, 38:191-201.

Pessenda, L.C.R; Gouveia, S.E.M; Aravena, R.; Boulet, R. \& Valencia, E.P.E. 2004. Holocene fire and vegetation changes in Southeastern Brazil as deduced from fossil charcoal and soil carbon isotopes. Quaternary International, 114:35-43. doi:10.1016/S1040-6182(03)00040-5

Pessenda, L.C.R.; Gouveia, S.E.M.; Freitas, H.A.; Ribeiro, A.S.; Aravena, R.; Bendasolli, A.; Ledru, M.P.; Siefeddine, A.F. \& Scheel-Ybert, R. 2005. Isótopos do carbono e suas aplicações em estudos paleoambientais. In: C.R.G. Souza; K. Suguio; A.M.S. Oliveira \& P.E. Oliveira (eds.) Quaternário do Brasil, Editora Holos, p. 75-90.

Pessenda, L.C.R.; Valencia, E.P.E.; Camargo, P.B.; Telles, E.C.C.; Martinelli, L. A.; Cerri, C.C.; Aravena, R. \& Rozanski, K. 1996 b. Natural radiocarbon measurements in Brazilian soils developed on basic rocks. Radiocarbon, 38:2:203-208.

Piperno, P. 1985. Phytolith analysis and tropical paleo-ecology: production and taxonomic significance of siliceous forms in new world plant domesticates and wild species. Review of Palaeobotany and Palynology, 45:185-228. doi:10.1016/00346667(85)90002-8
Piperno, D.R. 1988. A phytolith analysis: an archaeological and geological perspective. London, Academic Press, 280 p.

Piperno, D.R. 1991. The status of phytolith analysis in the American tropics. Journal of World Prehistory, 5:155-191. doi:10.1007/ bf00974678

Piperno, D.R. 2006. Phytoliths: a comprehensive guide for archaeologists and paleoecologists. Oxford, AltaMira Press, $238 \mathrm{p}$.

Piperno, D.R. \& Becker, P. 1996. Vegetacional history of a site in the central Amazon Basin derived from phytolith and charcoal records from natural soils. Quaternary Research, 45:202-209. doi:10.1006/qres.1996.0020

Piperno, D.R. \& Pearsall, D.M. 1998. The silica bodies of tropical American grasses: morphology, taxonomy, and implications for grass systematics and fossil phytolith identification. Washington, Smithsonian Institution Press, 44 p. (Smithsonian Contributions to Botany 85).

Raitz, E. 2012. Coleção de referência de silicofitólitos da flora do sudoeste do Paraná: subsídios para estudos paleoambientais. Programa de Pós-Graduação em Geografia, Universidade Estadual do Oeste do Paraná, Dissertação de mestrado, 204 p.

Rasbold, G.G.; Monteiro, M.R.; Parolin, M.; Caxambu, M.G. \& Pessenda, L.C.R. 2011. Caracterização dos tipos morfológicos de fitólitos presentes em Butia paraguayensis (Barb. Rodr.) L. H. Bailey (Arecaceae). Iheringia: Série Botânica, 66:265-270.

Rovner, I. 1988. Macro and micro-ecological reconstruction using plant opal phytolith data from archaeological sediments. Geoarchaeology, 3:155-163. doi:10.1002/gea.3340030207

Runge, F. 1999. The opal phytolith inventory of soils in central Africa-quantities, shapes, classification, and spectra. Review of Palaeobotany and Palynology, 107:23-53. doi:10.1016/s00346667(99)00018-4

Salgado-Labouriau, M.L. 1994. História Ecológica da Terra. $2^{\mathrm{a}}$ ed. São Paulo, E. Blücher, 307 p.

Sangster G. \& Parry, W.D. 1969. Some factors in relation to bulliform cell silicification in the grass leaf. Annals of Botany, 33:315-323.

Santos, G.M.; Alexandre, A.; Coe, H.H.G.; Reyerson, P.; Southon, R. \& Carvalho, C.N. 2010. The phytolith 14C puzzle: a tale of background determinations and accuracy tests. Radiocarbon, 52:113-128

Santos, J.C.A.; Gasparetto, N.V.L. \& Parolin, M. 2014. Reconstrução paleoambiental do baixo curso do rio Ivaí-Douradina/ Paraná. Geografia, 39:337-350.

Sendulsky, T.S. \& Labouriau, L.F.G. 1966. Corpos silicosos de gramíneas dos cerrados. I. Anais da Academia Brasileira de Ciências, 38:159-170.

Silva, D.W. 2013. Caracterização paleoambiental da região de Guarapuava-PR, a partir de sedimento de turfa: um estudo de caso. Programa de Pós-Graduação em Geografia, Universidade Estadual do Cetro-Oeste, Dissertação de mestrado, 90 p.

Suguio, K. 1999. Recent progress in Quaternary geology of Brazil. Episodes, 22:2217-220.

Twiss, P.C. 1992. Predicted world distribution of C3 and C4 grass phytoliths. In: G. Rapp Jr. \& S.C. Mulholland (eds.) Phytolith systematics. Emerging issues, Plenum Press, p. 113-128.

Twiss, P.C.; Suess, E. \& Smith, R.M. 1969. Morphological classification of grass phytoliths. Soil Science Society of America Proceedings, 33:109-115.

Received in November, 2014; accepted in June, 2016. 